\title{
TNFR2 induced priming of the inflammasome leads to a RIPK1-dependent cell death in the absence of XIAP
}

\author{
Janin Knop ${ }^{1}$, Lisanne M. Spilgies ${ }^{1}$, Stefanie Rufli ${ }^{1}$, Ramona Reinhart ${ }^{2}$, Lazaros Vasilikos ${ }^{1}$, Monica Yabal ${ }^{3}$, Erika Owsley ${ }^{4}$, \\ Philipp J. Jost $\mathbb{D}^{3}$, Rebecca A. Marsh ${ }^{4}$, Harald Wajant ${ }^{5}$, Mark D. Robinson', Thomas Kaufmann $\mathbb{E}^{2}$ and \\ W. Wei-Lynn Wong (iD
}

\begin{abstract}
The pediatric immune deficiency X-linked proliferative disease-2 (XLP-2) is a unique disease, with patients presenting with either hemophagocytic lymphohistiocytosis $(\mathrm{HLH})$ or intestinal bowel disease (IBD). Interestingly, XLP-2 patients display high levels of IL-18 in the serum even while in stable condition, presumably through spontaneous inflammasome activation. Recent data suggests that LPS stimulation can trigger inflammasome activation through a TNFR2/TNF/TNFR1 mediated loop in xiap ${ }^{-1-}$ macrophages. Yet, the direct role TNFR2-specific activation plays in the absence of XIAP is unknown. We found TNFR2-specific activation leads to cell death in $x^{2} a^{-1-}$ myeloid cells, particularly in the absence of the RING domain. RIPK1 kinase activity downstream of TNFR2 resulted in a TNF/TNFR1 cell death, independent of necroptosis. TNFR2-specific activation leads to a similar inflammatory NF-kB driven transcriptional profile as TNFR1 activation with the exception of upregulation of NLRP3 and caspase-11. Activation and upregulation of the canonical inflammasome upon loss of XIAP was mediated by RIPK1 kinase activity and ROS production. While both the inhibition of RIPK1 kinase activity and ROS production reduced cell death, as well as release of IL-1 $\beta$, the release of IL-18 was not reduced to basal levels. This study supports targeting TNFR2 specifically to reduce $\mathrm{IL}-18$ release in XLP-2 patients and to reduce priming of the inflammasome components.
\end{abstract}

\section{Introduction}

Full length tumor necrosis factor (TNF) is a membrane bound protein, where the extracellular domain can be cleaved by TNF converting enzyme (TACE) to release a soluble form ${ }^{1}$. Soluble and membrane TNF can bind and activate TNF receptor 1 (TNFR1) while only the membrane bound form triggers TNFR2 activation ${ }^{2}$. The outcome of TNF/TNFR1 signaling can range from production of other cytokines, proliferation, survival and differentiation. While activation of TNFR1 does not normally lead to

\footnotetext{
Correspondence: W Wei-Lynn Wong (wong@immunology.uzh.ch) ${ }^{1}$ Institute of Experimental Immunology, University of Zürich, Zürich, Switzerland

${ }^{2}$ Institute of Pharmacology, University of Bern, Bern, Switzerland Full list of author information is available at the end of the article. These authors contributed equally: Janin Knop, Lisanne M. Spilgies Edited by H.-U. Simon
}

cell death, the capacity for TNFR1 to induce apoptosis or necroptosis is swayed by the ubiquitylation and phosphorylation of RIPK1 and the activation of pro-survival signals mediated by NF-kB and MAPK pathways ${ }^{3-5}$. Activation of complex II is blocked by cFLIP, which is transcribed upon TNF/TNFR1 activation, preventing caspase- 8 activity. In the absence or inhibition of caspase- 8 activity, necroptosis ensues through RIPK1 kinase activity, RIPK3 and MLKL necrosome activity ${ }^{6,7}$. Phosphorylation of MLKL causes a conformational change, allowing for pore formation and the release of intracellular components, as well as damage-associated molecular patterns (DAMPs) ${ }^{8}$.

The expression of TNFR2 on cells of the immune system and endothelial cells is highly regulated. Upon

\section{(c) The Author(s) 2019}

(c) (i) Open Access This article is licensed under a Creative Commons Attribution 4.0 International License, which permits use, sharing, adaptation, distribution and reproduction cc) in any medium or format, as long as you give appropriate credit to the original author(s) and the source, provide a link to the Creative Commons license, and indicate if changes were made. The images or other third party material in this article are included in the article's Creative Commons license, unless indicated otherwise in a credit line to the material. If material is not included in the article's Creative Commons license and your intended use is not permitted by statutory regulation or exceeds the permitted use, you will need to obtain permission directly from the copyright holder. To view a copy of this license, visit http://creativecommons.org/licenses/by/4.0/. 
binding of membrane-bound TNF to TNFR $2^{9}$ a complex is formed that consists of TRAF2, cIAP1, cIAP2 and TRAF ${ }^{10}$. The activation leads to the degradation of cIAP1 and TRAF2, and signals through the noncanonical NF-kB pathway ${ }^{11}$. Due to the absence of a death domain, TNFR2 is considered to be mainly involved in survival and maturation of immune cells. Yet, previous data suggest that in some tumor cell lines, TNFR2 can regulate cell death by the loss of cIAP1 and TRAF2, causing production of TNF and thereby TNFR1 activation ${ }^{12}$. In addition, immortalized macrophages died in response to TNFR2 via necroptosis when caspase activity was inhibited ${ }^{13,14}$.

In response to toll-like receptor (TLR) activation, XIAP deficient myeloid cells undergo TNF-dependent cell death, resulting in the activation of the inflammasome through RIPK3/caspase-8/caspase- $1^{15-17}$. The stimulation of TLRs upregulate inflammasome components such as NLRP3, thus priming the cell ${ }^{18,19}$. A secondary stimulus is required to activate the inflammasome in vitro. When caspase- 1 is cleaved and activated, downstream targets IL$1 \beta$ and gasdermin D are cleaved. Cleaved gasdermin D forms a pore like structure that facilitates the release of cleaved IL-1 $\beta$, IL-18 and the osmotic lysis of the cell ${ }^{20,21}$. In response to TLR stimulation, $x_{i a p}{ }^{-/}$macrophages showed increased inflammasome activation compared to wildtype through the activation of TNFR2 and subsequent degradation of cIAP1/TRAF2 ${ }^{22}$.

We sought to understand what function TNFR2 played in the absence of XIAP when normally it would not be expected to cause cell death in primary macrophages. Specific TNFR2 stimulation alone in XIAP deficient macrophages resulted in cell death due to the lack of E3 ligase activity. RIPK1 kinase activity was required for both the TNF production from TNFR2 stimulation, as well as the TNFR1 mediated cell death. Surprisingly, neither necroptosis nor apoptosis occurred in comparison to previously published results $^{13,22}$. Instead, we found that TNFR2 activation acts as a signal 1 for priming the inflammasome in primary macrophages independent of genotype, and the combination of XIAP loss and TNFR1 activation plays a role as signal 2 for activation. Interestingly, cell death was blocked by RIPK1 kinase inhibitor as well as reactive oxygen species (ROS) scavengers, and while many of the pro-inflammatory cytokines returned to baseline, IL18 required genetic mutation of RIPK1 kinase to reduce to baseline levels. Thus, our work separates a key cytokine implicated in the etiology of XIAP deficient patients (X linked lymphoproliferative disease 2; XLP-2) from cell death. Taken together, we discovered a novel role of XIAP to inhibit TNFR2 induced inflammatory pyroptosis, adding additional complexity to treat XLP-2 patients.

\section{Results}

\section{Loss of XIAP RING domain sensitizes myeloid cells to TNFR2 induced cell death}

Similar to others, we found recombinant mouse TNF alone caused cell death in XIAP deficient macrophages $^{15,23}$ (Fig. 1a). To assess if specific activation of TNFR1 or TNFR2 was responsible, we used human TNF (TR1-TNF) to activate only TNFR1 and the published nonameric mouse TNF fusion protein, $\mathrm{TNC}-\mathrm{sc}(\mathrm{mu})$ TNF80 (TNC-TNF), to specifically activate TNFR2 $2^{24}$. Specificity of these ligands was confirmed by using $\operatorname{tnfr} 1^{-1-}$ tnfr $2^{-/-}$fibroblasts with re-introduced TNFR1 or TNFR2. Briefly, the cytoplasmic portion of human TNFR1 or TNFR2 was replaced with Fas and activation of TNFR1 or TNFR2, with the respective ligands led to cell death ${ }^{25}$ (Fig. S1A-B). Western blots for NF-kB signaling (activation of p-p65 or p100 cleavage) were performed on $\operatorname{tnfr} 1^{-/-}$ and $\operatorname{tnfr} 2^{-/-}$macrophages to confirm specificity in macrophages (Fig. S1C). Bone marrow derived macrophages (BMDMs) from wildtype, $x_{i a p}{ }^{-/}$, ciap $^{-/-}$and ciap2 $2^{-/-}$ mice were treated with either TR1-TNF or TNC-TNF overnight and assayed for cell death (Fig. 1b). Interestingly, $x i a p^{-/-}$macrophages but not ciap $1^{-/-}$or ciap $2^{-/-}$ macrophages were sensitive to TNFR2 induced cell death but insensitive to TNFR1 stimulation. The same was seen when we stimulated freshly isolated macrophages $\left(\mathrm{CD} 11 \mathrm{~b}^{+} \mathrm{F} 4 / 80^{+}\right)$from $x_{i a p^{-1-}}$ bone marrow (Fig. S1D). To determine the kinetics of the observed cell death, BMDMs were imaged using time-lapse photography for the uptake of PI. Xiap ${ }^{-1-}$ macrophages started to die by $8 \mathrm{~h}$ after TNC-TNF treatment as shown by the increase in PI positivity compared to wildtype cells (Fig. 1c and S1E). Using xiap ${ }^{\Delta R I N G}$ macrophages ${ }^{26}$, we found the E3 ligase activity required for resistance to TNC-TNF induced cell death (Fig. 1d).

Various mutations in the coding region of XIAP have been identified, contributing to immune hyper-activation and tissue inflammation in XLP-2 patients ${ }^{27}$. To determine whether BIR/caspase binding or RING/E3 ligase activity in identified human XIAP mutations could be associated with TNFR2 induced cell death, we utilized the HoxB8 progenitor system with re-introduced identified patient mutations ${ }^{17}$. Induced HoxB8 expression retains the cells in a myeloid progenitor-like state and removal of HoxB8 expression results in granulocyte differentiation. Both wildtype and $x i a p^{-1-}$ HoxB8 progenitor cells were insensitive to TNFR2-induced cell death similar to previous reports ${ }^{13}$ (Fig. S1F). To assess the contribution of either the BIR or RING domain of XIAP to the observed cell death, XIAP constructs containing mutations found in XLP-2 patients were re-introduced into the HoxB8 progenitor cells and expression levels assessed by western blotting $^{17}$ (Fig. 1e). Differentiated $x_{i a p^{-/-}}$granulocytes were sensitive to TNFR2 induced cell death but not to 

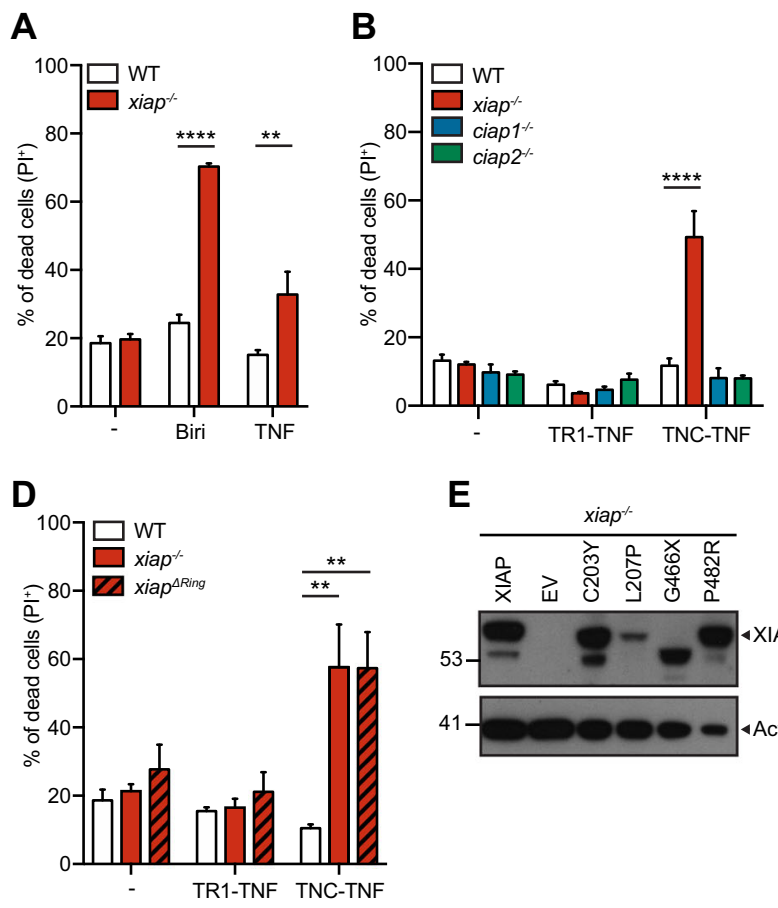

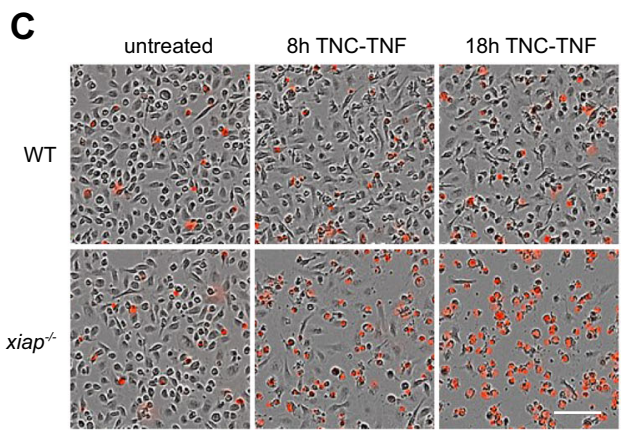

$\mathbf{F}$

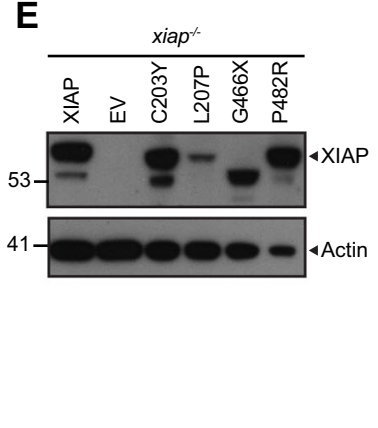

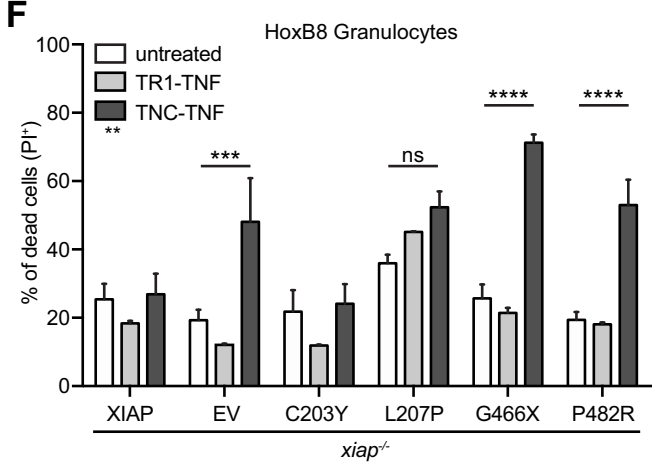

Fig. 1 Loss of XIAP results in TNFR2-mediated cell death in myeloid cells. $\mathbf{a}, \mathbf{b}, \mathbf{d}, \mathbf{f}$ Cell death was measured by the uptake of propidium iodide (PI) and analyzed by flow cytometry. a BMDMs from wildtype (WT) and xiap ${ }^{-/-}$were stimulated with Birinapant (Biri, $500 \mathrm{nM}$ ) and recombinant

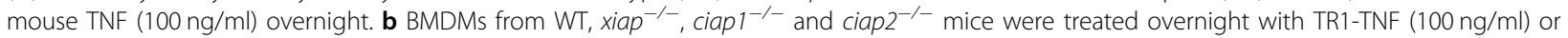
TNC-TNF $(100 \mathrm{ng} / \mathrm{ml})$. c Representative phase-contrast images merged with Pl positive images at 0,8 and $18 \mathrm{~h}$ after TNC-TNF stimulation (3 independent experiments performed). d BMDMs from WT, xiap ${ }^{-1-}$ and xiap ${ }^{\Delta-/-}$ mice were treated overnight with TR1-TNF or TNC-TNF. e Basal expression levels of XIAP in HoxB8 progenitors were assessed by western blot. Blot is a representative of three independent experiments. $\mathbf{f}$ Xiap ${ }^{-/-}$ HoxB8 granulocytes were transfected with WT, XIAP or XIAP mutated constructs and stimulated with TR1-TNF or TNC-TNF for $24 \mathrm{~h}$. $\mathbf{a}, \mathbf{b}, \mathbf{d}$ Data shown are mean \pm SEM including $n=3-5$ biological replicates. Experiments were repeated at least three times independently or $\mathbf{f}$ a minimum of three independent experiments, including triplicates for each experiment. Statistical significance was calculated using (a, b, d) two-way ANOVA or (f) Student $t$-test with ${ }^{* *} p<0.01,{ }^{* *} p<0.001$ and ${ }^{* * *} p<0.0001$.

TR1-TNF, while re-introduction of XIAP into the cells reduced TNFR2 induced cell death (Fig. 1f). Intriguingly, C203Y mutation in the BIR2 domain reduced the sensitivity of HoxB8 granulocytes to TNC-TNF while G466X and P482R were unable to rescue TNFR2 induced cell death (Fig. 1f). The expression levels for L207 construct were low and subsequently, sensitivity to TNC-TNF induced death occurred but was not significant. These data suggest that activation of TNFR2 in the absence of XIAP, specifically the E3 ligase domain, results in cell death in the myeloid compartment.

\section{TNFR2 stimulation leads to soluble TNF production resulting in TNFR1 mediated cell death in $x^{-1 a p^{-/}}$ macrophages}

Our previous data and others suggested that the inhibition or loss of XIAP, cIAP1, and cIAP2 leads to TNF production which may lead to TNFR1 mediated cell death $^{13,23}$. To test if TNFR2 activation results in subsequent release of TNF and TNFR1 activation, we stimulated macrophages for $4 \mathrm{~h}$ with TNC-TNF and subsequently added anti-TNF $\alpha$ to neutralize any TNF being produced upon the stimulation. TNC-TNF induced cell death was reduced significantly $(\sim 30 \%)$ (Fig. 2a). In agreement, $\mathrm{xiap}^{-/-} \mathrm{tnf}^{-/-}$macrophages were resistant to TNC-TNF induced cell death (Fig. 2b). Co-loss of TNFR1 or TNFR2 with XIAP deficient macrophages shows both TNFR1 and TNFR2 is required for cell death mediated by TNC-TNF (Fig. 2c). To determine if soluble TNF was produced, supernatant transfers from wildtype and $x_{i a p^{-1-}}$ macrophages treated with TNC-TNF to TNFR1Fas mouse fibroblast cells (TNFR1-Fas MF) showed TNFR1 mediated cell death was induced by soluble TNF produced by XIAP deficient macrophages (Fig. S2A).

To determine if the loss of cIAP1 or TRAF2 sensitized to TNFR1 mediated cell death as previously shown ${ }^{22}$, we assayed for degradation of TRAF2 in response to TNCTNF over time in wildtype and $x i a p^{-/}$macrophages. Degradation of TRAF2 occurred by $4 \mathrm{~h}$ in both wildtype and xiap $^{-1-}$ macrophages (Fig. 2d). We subsequently 
A

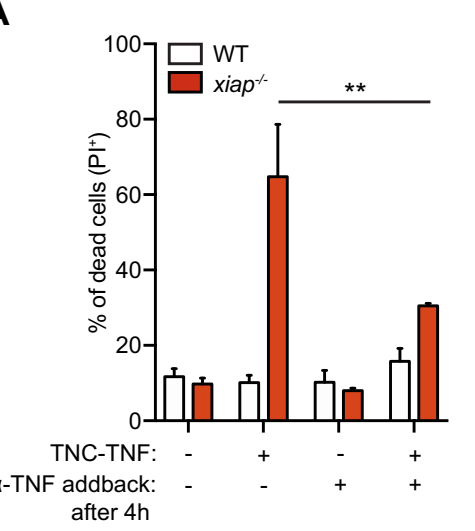

B

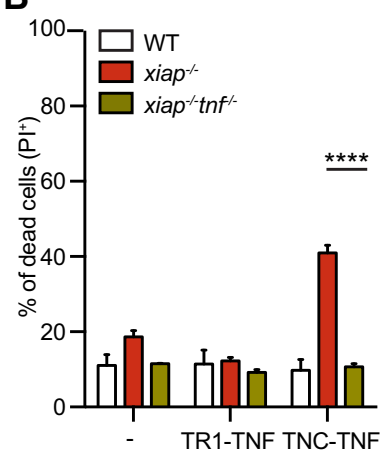

C

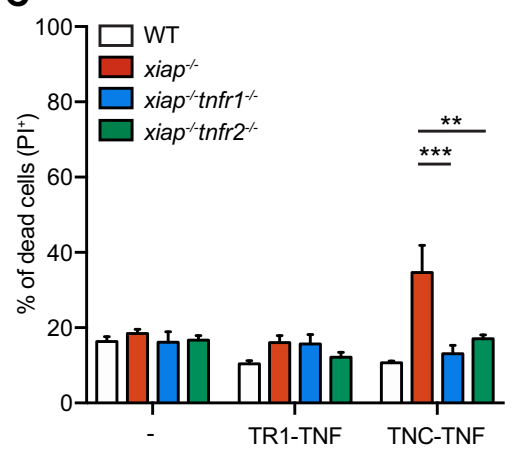

D
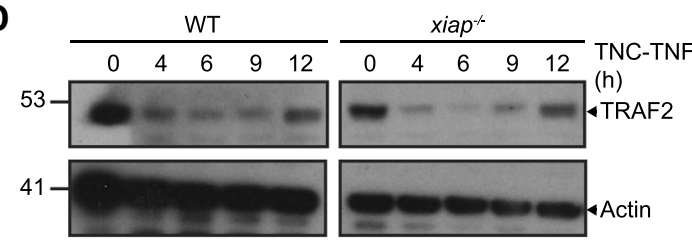

$\mathbf{F}$

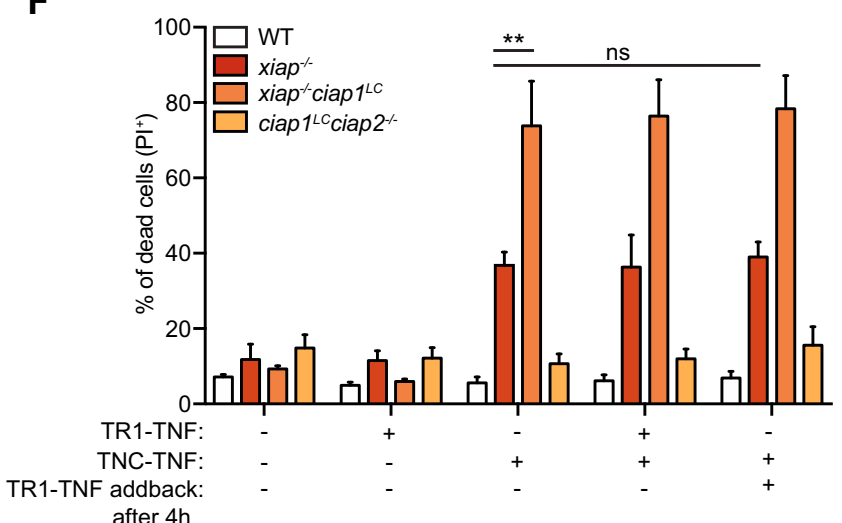

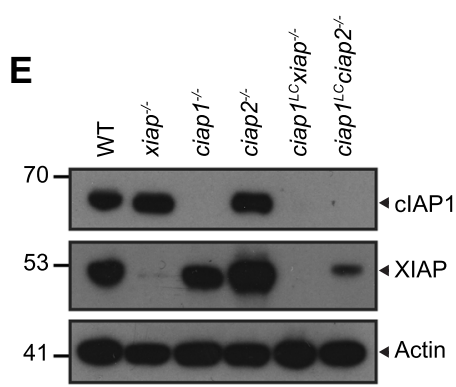

Fig. 2 TNFR2-induced cell death in xiap $^{-/-}$macrophages requires soluble TNF and TNFR1 activation. a BMDMs from WT and xiap ${ }^{-/-}$were treated with TNC-TNF and/or anti-TNFa $(100 \mathrm{ng} / \mathrm{ml})$ after $4 \mathrm{~h}$ of stimulation, and after $24 \mathrm{~h}$ cell death was measured via PI uptake by flow cytometry.

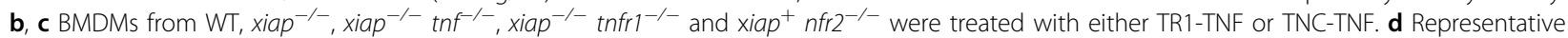
western blot shows that TRAF2 degrades in both WT and xiap ${ }^{-1-}$ BMDMs treated with TNC-TNF. e Representative western blot shows loss of clAP1 and XIAP in macrophage-specific (LC) genotypes. Blots are representative of three independent experiments. $\mathbf{f} W T, x_{i a p^{-/-}}, x_{i a p^{-/-}}$ciap $^{L C}$ and ciap 1 $^{\text {LC }}$ ciap2 $^{-1-}$ BMDMs were treated with TR1-TNF, TNC-TNF, either a combination of TR1- and TNC-TNF or an initial stimulation with TNC-TNF for $4 \mathrm{~h}$ and subsequent TR1-TNF stimulation. Data shown are mean \pm SEM including $n=3-5$ biological replicates. Experiments were repeated at least three times independently. Statistical significance was calculated using two-way ANOVA with ${ }^{* *} p<0.01,{ }^{* *} p<0.001$ and ${ }^{* * *} p<0.0001$.

treated wildtype, xiap $^{-/-}$, xiap ${ }^{-/-}$ciap1 $^{\text {LysMcre }}\left(\right.$ xiap $^{-/-}$ ciap $\left.^{L C}\right)$ or ciap $1^{L y s M c r e}$ ciap $2^{-/-}\left(\right.$ciap $\left.{ }^{L C}{ }^{\text {ciap }} 2^{-/-}\right)$macrophages with TR1-TNF and/or TNC-TNF. Interestingly, despite sufficient loss of cIAP1 in $\operatorname{ciap} 1^{L C}{ }_{\text {ciap }}{ }^{-/-}$and xiap ${ }^{-1-}$ ciap $^{L C}$ macrophages (Fig. 2e), neither genotypes were sensitive to TR1-TNF specific cell death. Preincubation with TNC-TNF for $4 \mathrm{~h}$ and subsequent stimulation with TR1-TNF did not sensitize wildtype cells to cell death nor did it trigger or enhance cell death in $\operatorname{ciap}^{L C-}$ ciap $2^{-1-}$ and $x i a p^{-1-} \operatorname{ciap}^{L C}$ macrophages, respectively
(Fig. 2f). These results suggest that TNFR2 specific activation initiates a cell death via TNF/TNFR1 in the absence of XIAP but the reduction of cIAP1/TRAF2 is not sufficient to trigger TNF/TNFR1 mediated cell death.

\section{TNFR2 mediated cell death in XIAP deficient macrophages} is RIPK1 kinase activity-dependent but independent of downstream necroptotic machinery or apoptosis

To determine the activity of caspases in TNFR2 mediated cell death, macrophages were treated with TNC-TNF and 
pancaspase inhibitors, QVD or ZVAD-fmk. TNFR2 induced cell death in $x i a p^{-1-}$ macrophages was slightly enhanced in the presence of QVD, suggesting cells switched from apoptosis to necroptosis, but no sensitization of wildtype cells to TNFR2 mediated cell death was seen. By contrast, use of ZVAD-fmk sensitized wildtype macrophages to TNFR2 mediated cell death as previously reported $^{13}$ (Fig. 3a). These results suggest the type of cell death mediated in the absence of XIAP is mechanistically different to combined use of ZVAD and TNC-TNF ${ }^{13}$. In agreement, no caspase-3 activity upon TNC-TNF stimulation was detected (Fig. S3A) ${ }^{23}$. To genetically confirm if necroptosis occurs, $x_{i a p}{ }^{-1-}$ ripk $^{-/-}$and $x i a p^{-1-}$ mlkl $^{-1-}$ macrophages were treated with TNC-TNF and imaged over time (Fig. 3b and Fig. 3SB). Loss of RIPK3 or MLKL in XIAP deficient cells did not rescue macrophages from TNFR2 mediated cell death. Furthermore, co-incubation with QVD did not rescue $x i a p^{-1}$ ripk $^{-1-}$ or $x i a p^{-1-}$ $m l k l^{-1-}$ from TNFR2 mediated cell death, suggesting no switch from apoptosis to necroptosis or vice versa when one pathway is blocked (Fig. 3c). However, co-incubation with RIPK1 kinase inhibitor (Necrostatin-1s, Nec-1s) entirely rescued TNC-TNF induced cell death. Even after $4 \mathrm{~h}$ of pre-stimulation with TNC-TNF, the inhibitor significantly rescued cell death (Fig. 3d). We further confirmed our findings using macrophages from xiap ${ }^{-1-}$ ripk $1^{\text {K45A/K45A }}\left(\right.$ xiap $^{-1-}$ ripk $\left.^{K D / K D}\right)$, a kinase inactive mutant $^{28}$. Consistent with Necrostatin-1s, macrophages from $x_{i a p^{-1}}$ ripk $^{K D / K D}$ mice were insensitive to TNCTNF induced cell death (Fig. 3e). Similarly, HoxB8 granulocytes were resistant to TNC-TNF in the presence of Nec1s (Fig. 3f). Using transmission electron microscopy, we identified cell membrane loss and limited membrane blebbing in $x_{i a p}{ }^{-1}$ macrophages treated with TNC-TNF suggesting that the cells were dying in a lytic fashion. Taken together, TNFR2 induced cell death in the absence of XIAP is dependent on RIPK1 kinase activity in myeloid cells.

\section{TNFR2 acts as a signal 1 in inflammasome activation}

To determine if XIAP influences downstream signaling of TNFR2, we probed for changes in NF- $\mathrm{KB}$ and MAPK pathways. In $x i a p^{-1-}$ macrophages, phosphorylation of p65 (p-p65) was prolonged compared to wildtype macrophages. Prolonged phosphorylation of p65 was also detected in $x i a p^{-/}$tnfr $^{-/-}$treated with TNC-TNF while $x i a p^{-/}$tnfr $2^{-/-}$macrophages did not show any activation of NF-kB and MAPK pathways, further confirming the specificity of TNC-TNF for TNFR2 and that the signaling changes are independent of TNFR1 (Fig. 4a). To determine if the sustained p65 signaling altered transcription in a XIAP specific manner, we profiled wildtype, xiap ${ }^{-/-}$and xiap ${ }^{-/-} \operatorname{tnfr} 1^{-/-}$macrophages treated with TNC-TNF for $2 \mathrm{~h}$. Comparison of the untreated samples of each genotype showed the gene expression was surprisingly similar (Fig. $4 \mathrm{~b}$ and Supp Table 1). Upon TNC-TNF stimulation, there were 88 genes uniquely regulated by the loss of XIAP, 47 in wildtype and 32 genes in $x i a p^{-1}$ tnfr $1^{-1-}$ macrophages. Only 15 genes were differentially regulated and overlapping between $x_{i a p^{-/-}}$and xiap ${ }^{-1-}$ tnfr $1^{-1-}$ macrophages when treated with TNC-TNF, suggesting changes in this set of genes was not influenced by TNFR1.

Gene ontology analysis showed an enrichment of genes involved in inflammation and cell death processes in a similar expression pattern independent of genotype (Fig. S4A). Surprisingly, gene set enrichment analysis showed that the differentially regulated genes upon TNFR2 stimulation were alike to those associated with TNF/TNFR1, particularly genes requiring NF-kB (Fig. 4c and Supp Table 2). Genes upregulated or downregulated in LPS gene signatures and KEGG NOD-like receptor signaling were also significantly enriched in our gene sets showing the cross over of pathways linking LPS and TNF induction (Supp Table 2). These data surprisingly show that XIAP loss was a minor influence in TNFR2-induced transcriptional changes but that TNFR2 itself is a driver for inflammation in macrophages.

Consistent with others ${ }^{14}$, we also found, tnfaip3 (A20) and traf1 upregulated by TNC-TNF in our RNAseq analysis (Fig. 4d and Supp Table 1). The anti-apoptotic response of cflar (c-FLIP) and birc3 (cIAP2) is consistent in $x_{i a p}{ }^{-1}$ and $x i a p^{-1-}$ tnfr1 $1^{-/}$macrophages (Fig. 4d). The log fold change plots show that while tnf, $c x c l 1, c x c l 2$, and $c c l 3$ are differentially regulated at the RNA level in all genotypes, there is a slight increase in RNA levels in the absence of XIAP or co-loss with TNFR1 (Fig. 4d, coded in black). $C c l 4$ and $i l-1 \beta$ were exclusively upregulated in $x_{i a p}{ }^{-1}$ macrophages (Fig. 4d, coded in gray). These data suggest that while TNFR2 directly stimulates the expression of key cytokines and chemokines, the cytokine network is further enhanced by the absence of XIAP and the stimulation of TNFR1. Interestingly, our analysis showed that in all wildtype, $x i a p^{-1-}$ and xiap ${ }^{-/}$tnfr $1^{-/-}$macrophages, TNFR2 stimulation induced inflammasome related components such as NLRP3 and caspase-11 (casp4). These results suggest that TNFR2 activation not only acts as a signal 1 for inflammasome priming but also induces the expression of cytokines and chemokines similar to TNFR1.

To confirm the involvement of the pyroptotic components in TNFR2 induced cell death, we treated WT, xiap $^{-/-}$, xiap ${ }^{-/}$tnfr $1^{-/-}$and $x i a p^{-/-}$tnfr $2^{-/-}$macrophages with TNC-TNF overnight and assayed the supernatant as well as the lysate for inflammasome activation. We found that indeed upon TNC-TNF stimulation, NLRP3 and caspase-11 were upregulated (Fig. 4e). 


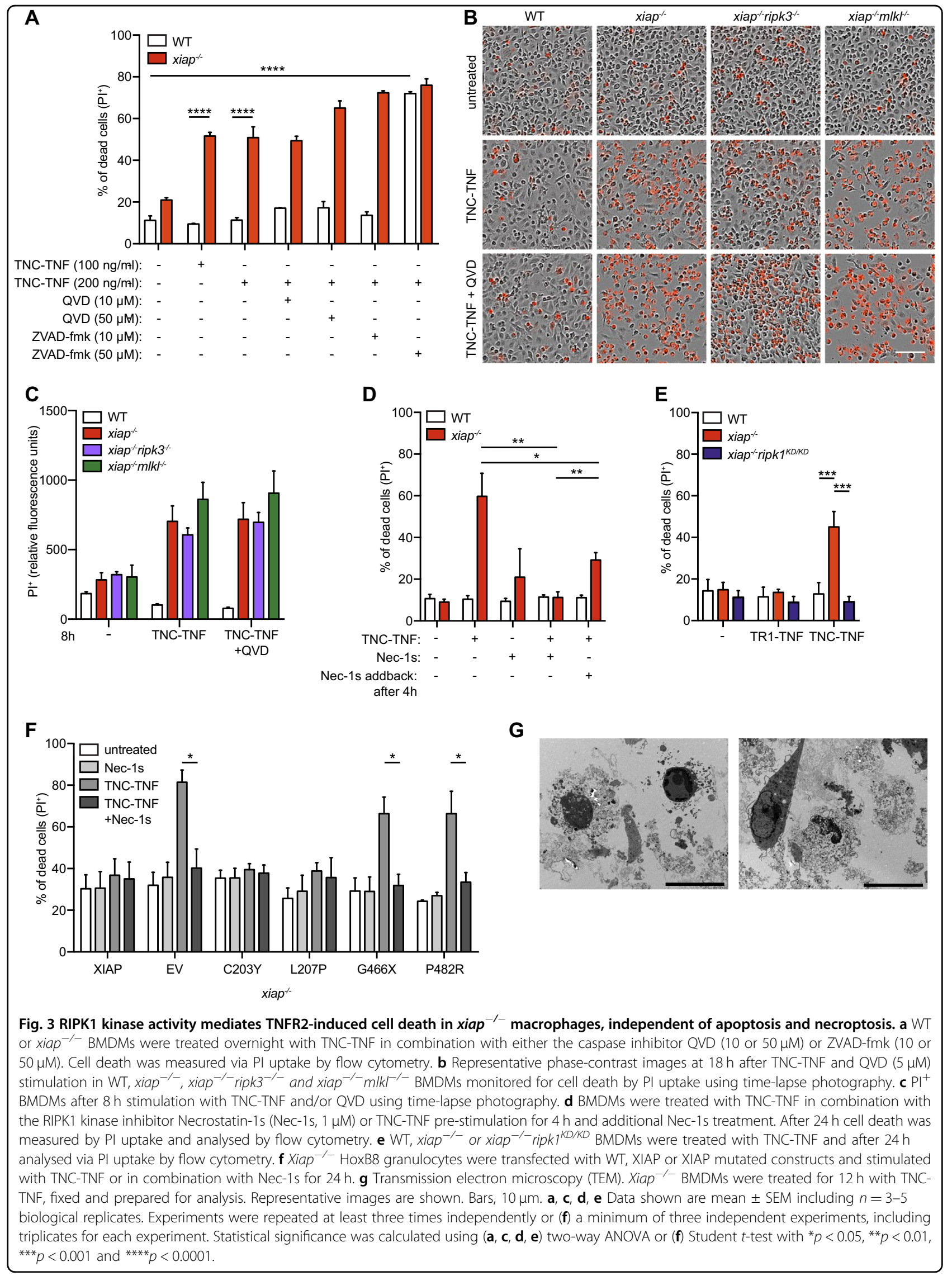




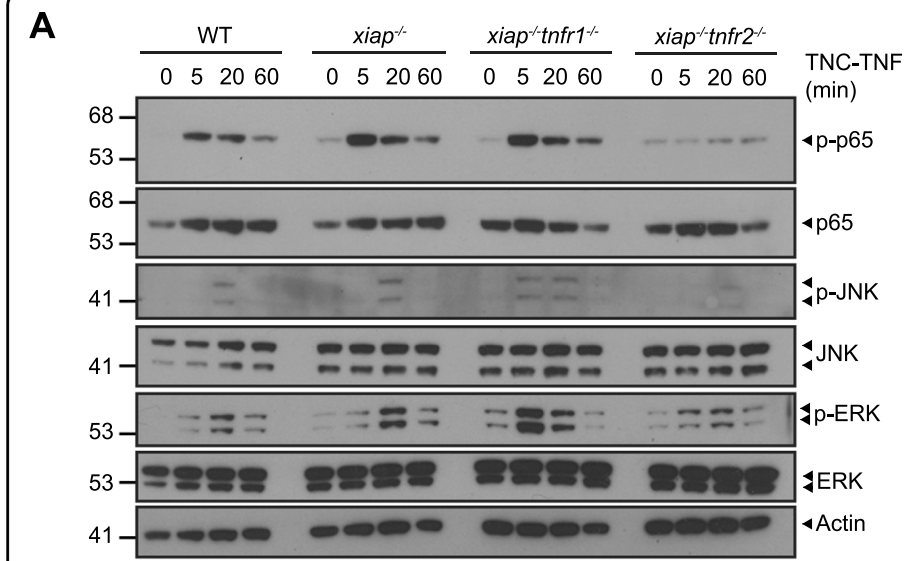

C

Tian TNF signalling via NFKB (21)

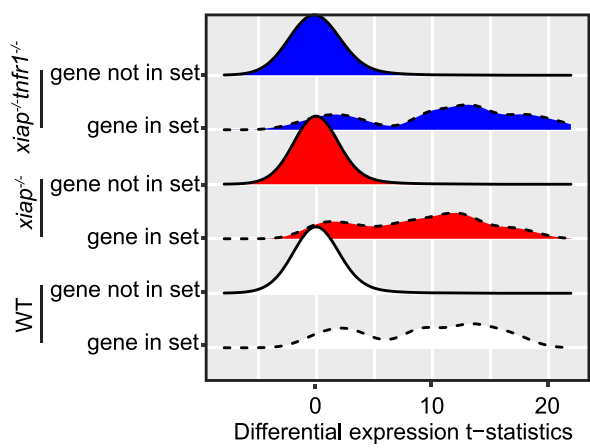

D

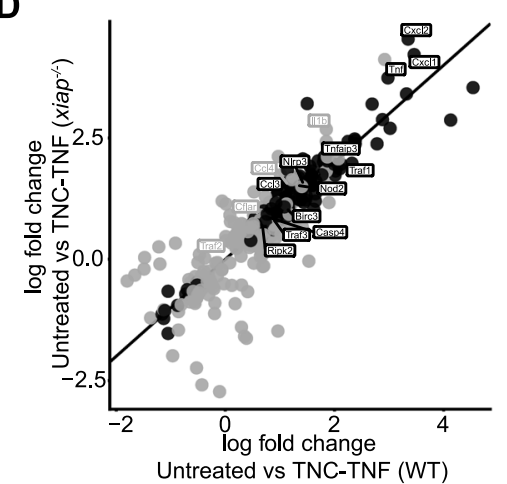

Tian TNF signalling not via NFKB (20)

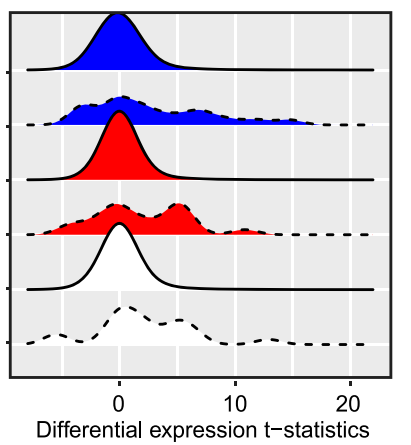

B
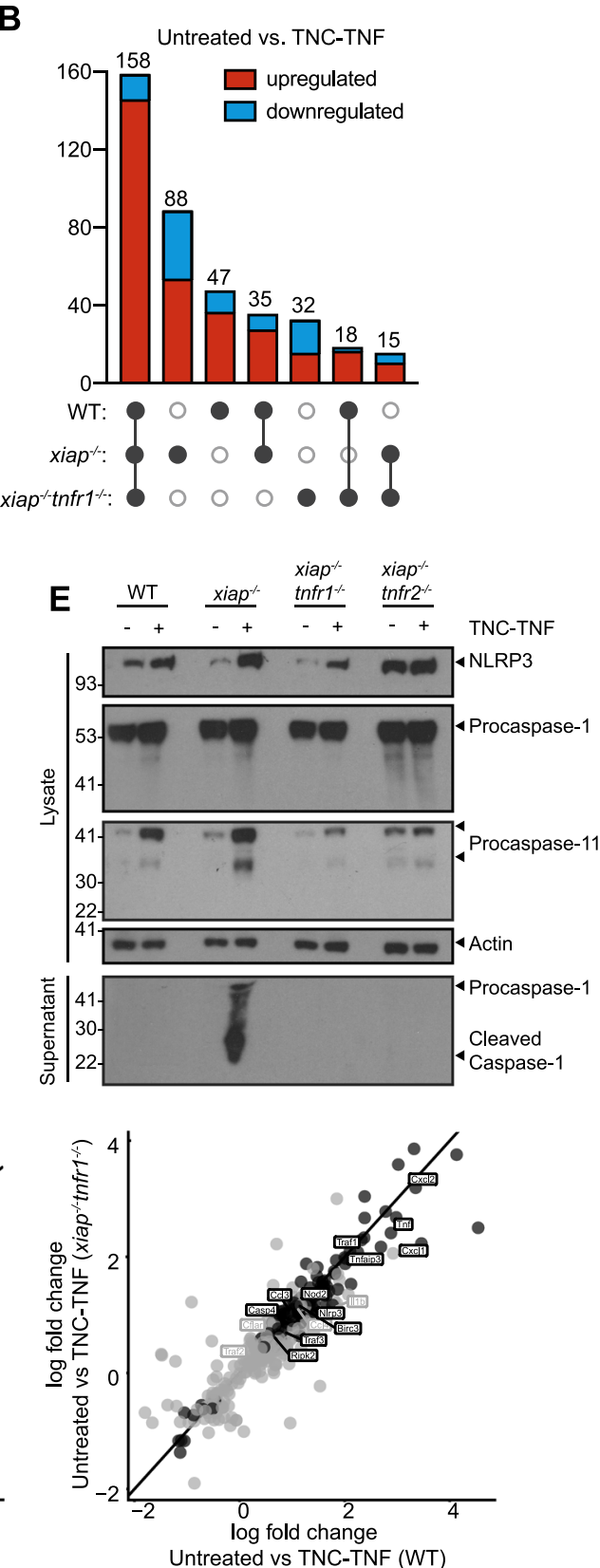

Fig. 4 TNFR2 primes inflammasome components. a BMDMs were treated with TNC-TNF for the indicated time points and expression levels of proteins of the NF-KB and MAPK signaling pathway were analysed by western blotting. Blots are representative of three independent repeats. b Upset plot representing the detected changes in expression between untreated and TNC-TNF treated for each of the genotypes, including up- and downregulated genes. c Gene set analysis of Tian TNF signalling via NF-kB or not via NF-KB (number of genes in the gene set are given in parentheses). The distributions of differential expression $t$-statistics (computed by voom) are shown for the genes within the gene set and not within the gene set for each genotype. $\mathbf{d}$ Comparison of differentially regulated genes in WT, xiap ${ }^{-1-}$ and xiap ${ }^{-/-}$tnfr $^{-1-}$ macrophages after TNC-TNF treatment. Black dots represent genes that are differential at FDR $<0.05$ in all three genotypes, and grey dots represent genes that are differential at FDR $<0.05$ in two genotypes. e Representative western blot showing TNC-TNF treatment leads to upregulation of NLRP3, caspase-11 and procaspase-1, but only in xiap $^{-1-}$ macrophages is caspase-1 cleavage detected. Blots are representative of three independent experiments.

However, the activation of caspase- 1 and cleavage of gasdermin D was only observed in XIAP deficient cells. The increase in gasdermin D, caspase- 1 and NLRP3 by
TNC-TNF was independent of TNFR1. These results suggest TNFR2 activation alone can act as a signal 1 in inflammasome activation. 


\section{Pro-inflammatory cytokines induced by TNFR2 are dependent on TNF and RIPK1 kinase activity}

Since the RNAseq showed an increased enrichment of inflammation, we screened for cytokine and chemokine production. From this assay, we found several cytokines and chemokines further upregulated in $x_{i a p}{ }^{-/-}$macrophages compared to wildtype when treated with TNCTNF for $24 \mathrm{~h}$ (Fig. S4A). The key cytokines, IL-1 $\beta$, IL-18, and IL-6, implicated in symptoms of XLP-2 patients were identified as increased compared to treated wildtype macrophages ${ }^{29}$. Using xiap ${ }^{-/-}$tnf $^{-/}$, $x^{2 i a p^{-/-}} \operatorname{tnfr} 1^{-1-}$, and xiap $^{-1-}$ tnfr $2^{-1-}$ macrophages, we determined IL-1 $\beta$, IL-18, and IL-6 were dependent on TNF production and required both TNFR1 and TNFR2 present for the production of these cytokines (Fig. 5a).

Because cell death and inflammation are closely interwined, we asked whether inhibition of RIPK1 kinase activity would also reduce inflammation. The loss of RIPK1 kinase activity resulted in a reduction of TNF mRNA but only in the absence of XIAP (Fig. 5b). Protein levels of IL-1 $\beta$ and IL- 6 were easily reduced in response to RIPK1 kinase activity inhibition. However, pre-incubation and high dose of Nec-1s was required to reduce IL-18 (Fig. 5c). Using $x i a p^{-1-}$ ripk $1^{K D / K D}$ macrophages, IL-1 $\beta$, IL-6, and IL-18 production were completely reduced when treated with TNC-TNF (Fig. 6d). Similar to previous data with LPS stimulation ${ }^{15,30}$, we found RIPK3 loss reduced IL-1 $\beta$ but not IL-6 in $x i a p^{-/}$ripk $^{-/-}$and $x i a p^{-/-}$ $m l k l^{-1-}$ macrophages in response to TNC-TNF (Fig. S4B). These results imply direct TNFR2 activation in the absence of XIAP leads to increased pro-inflammatory IL$1 \beta$, IL-18, and IL-6 in a RIPK1 kinase-dependent manner.

Interestingly, the loss of RIPK1 kinase activity resulted in a slight decrease in gasdermin D and caspase-11 protein levels, as well as cleavage of gasdermin D suggesting the predominant role of RIPK1 kinase activity is to reduce the production of TNF in response to TNFR2 stimulation (Fig. 5e).

\section{XIAP restricts the activation of NLRP3 inflammasome (signal 2) by regulating ROS production}

Previous results suggest that ROS production may influence inflammasome activation. We assayed for ROS production and found in the absence of XIAP, an increased amount of ROS was detected which was reduced by the use of free radical scavengers, BHA or NAC (Fig. 6a). Co-incubation of TNC-TNF and BHA also reduced cell death in the $x i a p^{-1-}$ macrophages (Fig. 6b) and protein levels of NLRP3, gasdermin D and caspase-11 were also reduced and subsequent caspase- 1 cleavage was missing (Fig. 6c). ROS scavengers reduced IL- $1 \beta$ and IL-6 but IL-18 levels remained higher than baseline (Fig. 6d).

To determine the significance of caspase-1 and NLRP3 activation in the production of IL- $1 \beta$ and IL-18, we co- incubated cells with caspase-1 inhibitor (VX-765 or Ac YVAD-cmk) with TNC-TNF. The loss of caspase-1 activation resulted in reduced levels of IL-1 $\beta$, IL-18, and IL-6 in the supernatant of treated $x i a p^{-1-}$ macrophages (Fig. 6e). Using $x i a p^{-1-} n l r p 3^{-/-}$macrophages with xiap $^{-1-}$ on the Harlin et al. background ${ }^{31}$, we assayed for IL-1 $\beta$, IL-6, and IL-18 in response to TNC-TNF compared to the matched $x i a p^{-1-}$ strain. Surprisingly, the xiap $^{-1-31}$ did not die in response to TNC-TNF but a decrease in IL-1 $\beta$ was detected and IL-18 was not detected at all (Fig. 6f).

Finally, to determine if TNFR2 activation is sufficient as signal 1, we incubated cells with TNC-TNF and then treated with nigericin or ATP as a second signal. Gasdermin D and caspase-1 cleavage were detected in wildtype macrophages, as well as $x_{i a p^{-1-}}$ macrophages. However, in xiap ${ }^{-/}$tnfr $1^{-/-}$macrophages, no cleavage was detected (Fig. 6g). Taken together, in the absence of XIAP, increased ROS production leads to NLRP3 induced activation and release of IL-1 $\beta$.

\section{Discussion}

TNFR2 has been shown to promote survival, differentiation and induce immune suppressive functions ${ }^{32,33}$. However, our data suggest the opposite in the absence of XIAP. Here, we provide evidence that the activation of TNFR2 primes macrophages towards inflammasome activation with dominant expression of proinflammatory cytokines, as well as PRR sensors. Only in the absence of the E3 ligase activity of XIAP do myeloid cells die by pyroptotic activity in response to direct TNFR2 stimulation.

The concept of non-death domain TNF super family receptors (TNFSFR) involved in cell death has been previously reported in cancer cell lines for FN14, CD40, TNFR2, and CD30, acting through a TNF/TNFR1 axis $^{12,34,35}$. Our data differs from recently published results implicating TNFR2 in LPS-induced cell death in the absence of XIAP where necroptosis, pyroptosis and apoptosis is implicated $^{22}$. In the absence of XIAP, TNFR2 induced cell death does not appear to induce caspase activity, nor is there switching from apoptosis to necroptosis as $\mathrm{xiap}^{-1-} \mathrm{mlkl}^{-1-}$ and $\mathrm{xiap}^{-/} \mathrm{ripk}^{-1-}$ in the presence of QVD does not rescue cell death in response to TNFR2 activation. These data suggest that the inflammation may be a separable function to the cell death or preceding cell death activation. Caspase- 8 could serve as a scaffold for a NF-kB inducing complex that includes RIPK1 as proposed by Henry et al. to promote inflammation by TRAIL activation ${ }^{36}$. Our data supports this theory as TNFR2 specific activation leads to TNF mRNA production requiring RIPK1 kinase activity, and priming of both pro-inflammatory cytokines and chemokines is present in both wildtype and XIAP deficient cells. These data would suggest RIPK1 kinase activity can 


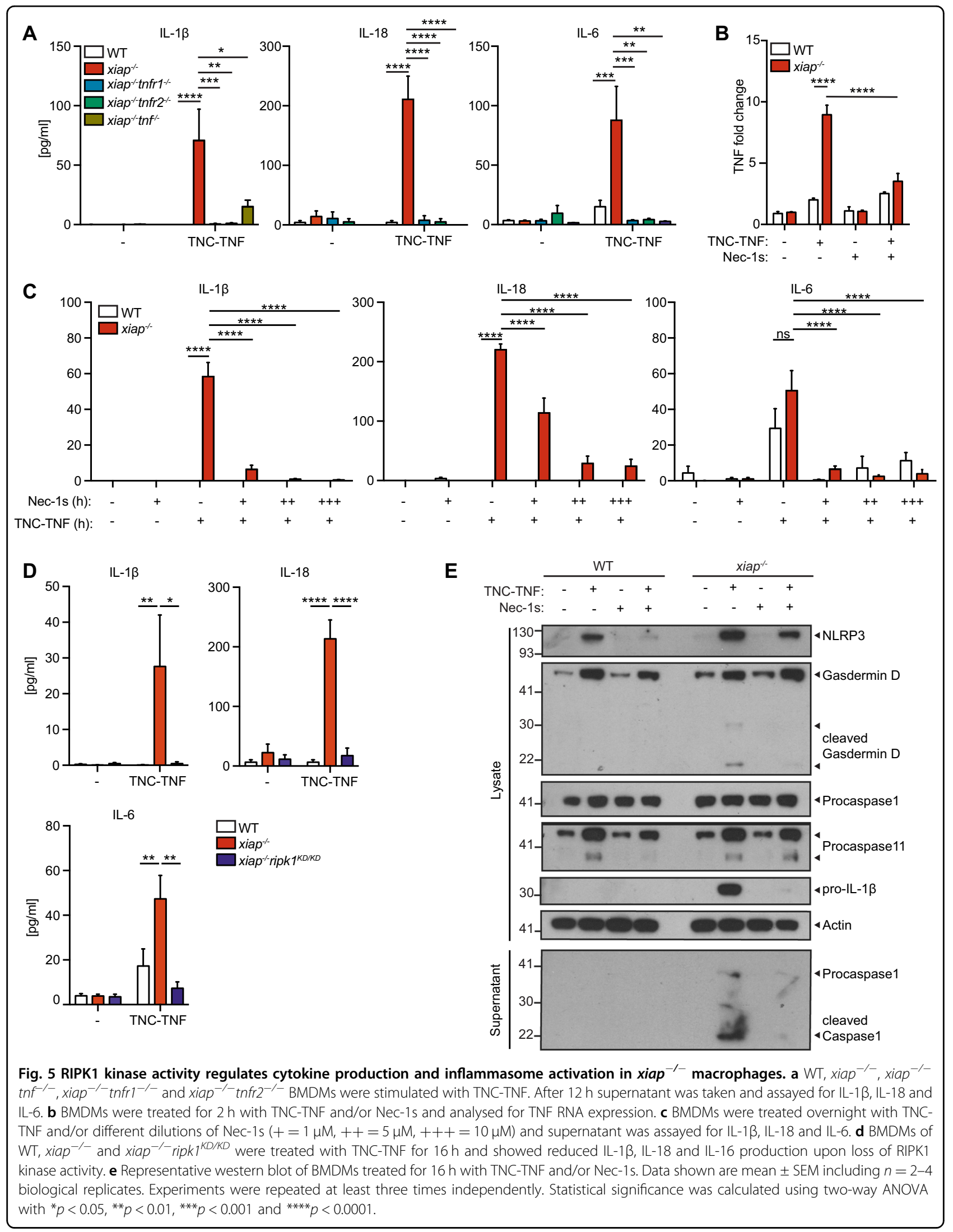




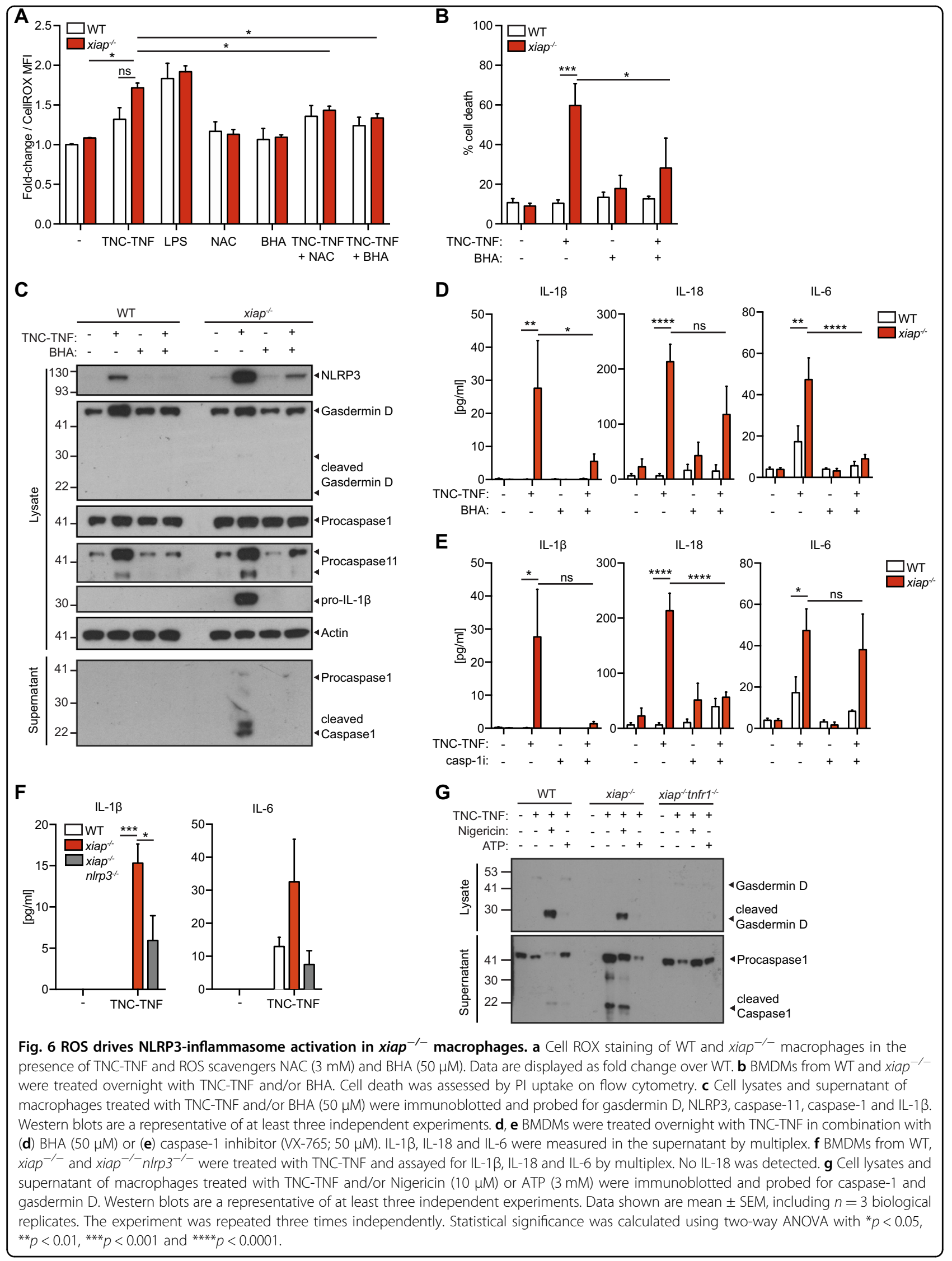


mediate inflammation, specifically TNF, in line with previous results linking the kinase activity of RIPK1 to inflammation in the absence of IAPs $^{23,28,37}$. However, further studies would be required to determine if complete inhibition of RIPK1 kinase activity would be achievable in vivo and be effective after inflammation is in progress.

TNFR2-specific activation mediated NOD2 and RIPK2 upregulation, suggesting that TNF may act as a primer for the detection of intracellular pathogens. Indeed, a lack in inflammation has been identified in XLP-2 patients in response to MDP/NOD2 activation through loss of RIPK2 ubiquitylation by XIAP and cIAPs $^{38-40}$. In response to NOD2 stimulation, both mutations in the BIR and RING domain led to a loss in NF-kB ${ }^{41}$. By contrast, our data shows that TNFR2 induced cell death occurs in the absence of E3 ligase activity of XIAP. Whether the loss of E3 ligase activity of XIAP relates to the ROS production is unknown. Further studies in identifying what substrate(s) of XIAP will prevent TNFR2 induced cell death would be beneficial in the treatment of XLP-2 patients.

Our findings are of therapeutic interest as XLP-2 patients have increased inflammasome related cytokines. XIAP deficient patients are characterized with multiorgan inflammation triggered by viral infections, as well as intestinal bowel disease ${ }^{42,43}$. XLP-2 is associated with activated macrophages and lymphocytes and overexpression of pro-inflammatory cytokines including TNF during an HLH episode ${ }^{29,44}$. During viral infections, the increased TNF presence would trigger TNFR1 and TNFR2. Our study provides evidence that inhibition of cell death may not result in reduction of key inflammasome cytokines such as IL-18 in XLP-2 patients and that there is differential regulation of IL- $1 \beta$ in comparison to IL-18 as recently published ${ }^{45}$. Contrary to most situations, targeting TNFR2 with blocking antibodies can be of potential therapeutic interest in XIAP deficient patients to limit inflammation.

\section{Materials and methods}

Mice

$\mathrm{Xiap}^{-/-}, \operatorname{ciap}^{-/-}, \operatorname{ciap} 2^{-/-}$, and $\operatorname{ciap}{ }^{L C}{ }^{L i a p} 2^{-/-}$mice were a kind gift from J. Silke from WEHI and were previously described ${ }^{46,47}$. These strains were embryo transferred and maintained in an SPF facility in Zurich. Tnfr $1^{-/-}$ and $t n f r 2^{-1-}$ mice were a kind gift from A. Fontana and A. Aguzzi, respectively ${ }^{48}$, and were crossed to $x_{i a p^{-1}}$ mice to generate $x_{i a p^{-/}}$tnfr $^{-/-}$and $x i a p^{-/}$tnfr $2^{-/}$mice. Ripk $1^{K 45 A / K 45 A}\left(r i p k 1^{K D / K D}\right)$ mice were a gift from GlaxoSmithKline and were crossed to $x_{i a p^{-/-}}$mice to generate xiap ${ }^{-1}$ ripk $^{K D / K D}$ mice. All mice used in this study were back-crossed to C57BL/6 mice. All animal experiments were performed at the University of Zurich under the ethical license 186/2015. Xiap $^{-/-}$and xiap $^{-1-}$ $n l r p 3^{-/-}$mice were a kind gift from RA Marsh ${ }^{31}$. Xiap ${ }^{-/-}$ $\mathrm{tnf}^{-/-}$, $\mathrm{xiap}^{-/-}$ripk $^{-/-}$, xiap ${ }^{-/} \mathrm{mlkl}^{-/-}$and $x_{i a p}{ }^{\Delta R I N G}$ mice were a kind gift from P. Jost and housed at the Technical University of Munich. Experiments were conducted in accordance with GSK policies on the care, welfare, and treatment of laboratory animals.

\section{Generation of bone marrow derived macrophages and cell lines}

To generate bone marrow-derived macrophages (BMDMs), bone marrow was obtained from the tibia and femur of 6-12-week-old mice. Cells were cultured on petri dishes for 5 days in Dulbecco's modified Eagle medium (DMEM, Gibco) containing $1 \mathrm{~g} / \mathrm{L}$ glucose, $1 \%$ (v/v) penicillin/streptomycin/glutamine (Gibco), 10\% FBS (SeraGlobe) and supplemented with 20\% (v/v) L929 mouse fibroblast conditioned medium. On day 5 , cells were harvested and seeded at $1 \times 10^{6}$ cells $/ \mathrm{mL}$ into the desired tissue culture plates (e.g., $1 \times 10^{5}$ cells per 96 well). TNFR1-Fas and TNFR2-Fas expressing mouse fibroblasts were obtained from Anja Krippner-Heidenreich ${ }^{25}$ and were cultured in DMEM containing $1 \mathrm{~g} / \mathrm{L}$ glucose, $1 \%$ (v/v) penicillin/streptomycin/glutamine and 10\% FBS. HoxB8 progenitor cells were cultured in RPMI 1640 media with $10 \%(\mathrm{v} / \mathrm{v})$ heat-inactivated FBS, $1 \%(\mathrm{v} / \mathrm{v})$ penicillin/streptavidin, 7\% (v/v) SCF from $\mathrm{CHO} / \mathrm{SCF}$ conditioned medium, and $0.1 \mu \mathrm{M}$ 4-hydroxytamoxifen (4-OHT, MedChemExpress) ${ }^{17}$. To differentiate granulocytes from the HoxB8 progenitors, cells were washed twice with PBS and re-suspended at a concentration of $2.5 \times 10^{4}$ cells $/ \mathrm{mL}$ in media without $4-\mathrm{OHT}$ and differentiated for 5 days.

\section{Ligands and inhibitors}

To produce the agonistic TNFR2 ligands, pCR3 Fc-FlagTNFR2-specific nonameric murine TNF variant (TNCTNF), trimeric pCR3 Flag-TNC-TNF or pCR3 Fc-Flag human TNFR1 (TNFR1-TNF), were transfected into 293t cells and purified as previous described ${ }^{49}$. Endotoxin levels were tested and removed (Pierce High Capacity Endotoxin Removal Spin columns, ThermoScientific). TNF variants were used at $100 \mathrm{ng} / \mathrm{mL}$. Inhibitors were used at the following concentrations: Birinapant $(500 \mathrm{nM}$, Chemietek), Compound A (100 nM, Tetralogics), multimeric TNF (mega TNF, $100 \mathrm{ng} / \mathrm{mL}$, Adipogen) Necrostatin-1s (1, 5 or $10 \mu \mathrm{M}$, MedChemExpress), Q-VDOPH (5, 10 or $50 \mu \mathrm{M}$, Adipogen), ZVAD-fmk (5, 10 or $50 \mu \mathrm{M}$, MedChemExpress), VX-765 (10 $\mu \mathrm{M}$, Invivogen), BHA (50 $\mu \mathrm{M}$, Sigma), Nigericin (10 $\mu \mathrm{M}$, Sigma) and ATP (3 mM, Sigma).

\section{Antibodies}

The following antibodies were used for flow cytometry: CD11b-PeCy7 (clone M1/70, eBioscience), F4/80 (clone 
BM8, eBioscience), and fixable viability dye (eBioscience). The following antibodies were used for western blotting: phospho-p65, NF-kB2, phospho-ERK, phospho-JNK, phospho-p38, total ERK, total JNK, and total p38 from Cell Signaling Technologies. Total p65 was purchased from Santa Cruz. TRAF2, gasdermin D and caspase-11 were purchased from Abcam, caspase- 1 from Adipogen and IL- $1 \beta$ from RnD. cIAP1 was purchased from Human Atlas. RIPK1 and XIAP was purchased from BD Biosciences. Secondary antibodies for western blotting such as donkey anti mouse/rabbit/rat IgG conjugated to HRP are from SouthernBiotec, the donkey anti goat IgG was purchased from Santa Cruz. The neutralizing antibody against TNF (at $200 \mathrm{ng} / \mathrm{mL}, \mathrm{MP6}-\mathrm{XT} 22$ ) was purchased from BioLegend.

\section{Cell death analysis}

Cells were seeded at a density of $1 \times 10^{6}$ cells $/ \mathrm{mL}$. After treatment, cells were trypsinized, washed once and resuspended in HBSS containing $1 \mu \mathrm{g} / \mathrm{mL}$ propidium iodide (PI) or stained with fixable viability dye (eBioscience). Cells were then assessed for cell death by flow cytometry on a FACS Canto II. Data were analyzed by FlowJo software, version 10.2. Alternatively, cells were directly incubated in the presence of $5 \mu \mathrm{g} / \mathrm{ml}$ propidium iodide and assessed for viability by acquiring both phase contrast and red fluorescence images at $2 \mathrm{~h}$ intervals at $\times 10 \mathrm{mag}$ nification over $24 \mathrm{~h}$ using the IncuCyte. Confluency and PI fluorescence were measured and analyzed using the IncuCyte Zoom Software (Version 2016A).

\section{Cell sorting}

To isolate primary macrophages from mouse bone marrow, $\mathrm{CD} 11 \mathrm{~b}^{+} \mathrm{F} 4 / 80^{+}$cells were separated using a FACSMelody $3 \mathrm{~L}$ machine.

\section{Caspase activity assay}

Cell lysates were treated and lysed in DISC lysis buffer $(20 \mathrm{mM}$ Tris- $\mathrm{HCl} \mathrm{pH} 7.5,150 \mathrm{mM} \mathrm{NaCl}, 10 \%$ (v/v) glycerol, $1 \%(\mathrm{v} / \mathrm{v})$ Triton X-100, with protease and phosphatase inhibitors) and incubated with $0.5 \mathrm{mM}$ DEVDAMC. Results were normalized to the protein concentration, that was calculated by BCA (Pierce) according to manufacturer's instructions.

\section{Multiplex cytokine analysis}

Multiplex cytokine analysis (ProcartaPlex, Thermo Scientific) was performed according to the manufacturer's instructions and assayed on a Bio-Rad Bioplex machine.

\section{Western blotting}

Supernatant was collected when indicated and precipitated using $4 \%$ trichloroacetic acid (TCA) before being pelleted in acetone, boiled and run like lysates described in the following. Cells were lysed using DISC lysis buffer (20 mM Tris- $\mathrm{HCl} \mathrm{pH} 7.5,150 \mathrm{mM} \mathrm{NaCl}, 10 \%$ (v/v) glycerol, 1\% (v/v) Triton X-100, with protease and phosphatase inhibitors). The insoluble fraction of the lysate was pelleted by centrifugation and removed. Lysates were boiled and run on $4-12 \%$ Bis-Tris Gel NuPAGE using MOPS buffer (Invitrogen). Proteins were then transferred onto PVDF-membrane $(0.2 \mu \mathrm{m}$, Thermo Scientific) using the Trans-Blot ${ }^{\circledR}$ Turbo $^{\text {tw }}$ Transfer System (Bio Rad) or the Pierce $^{\text {Tu }}$ Power Blotter (Thermo Scientific), both according to the manufacturer's instruction. After blocking with PBST containing $5 \%(\mathrm{w} / \mathrm{v})$ skim milk, membranes were incubated with the indicated primary antibody in either PBST containing $5 \%$ skim milk or $5 \%$ (w/v) BSA (Fraction V, Sigma) for at least $1 \mathrm{~h}$ at RT or overnight at $4{ }^{\circ} \mathrm{C}$. Protein level expression was acquired using WesternBright ECL (Advansta) and Amersham Hyperfilm ECL (GE Healthcare).

\section{qPCR}

RNA was isolated using GENEzol Reagent (Geneaid) according to the manufacturer's instructions. cDNA was produced using MultiScribe ${ }^{\mathrm{mm}}$ Reverse Transcriptase and SYBR Green qPCR master mix (Thermo Fisher Scientific) was used for running the qPCR. Melting curves showed that single products were formed. The following primers were used: TNF (5': CCA CCA CGC TCT TCT GTC TA; 3': CAC TTG GTG GTT TGC TAC GA); B2M (5': TGG TGC TTG TCT CAC TGA CC; $3^{\prime}$ CCG TTC TTC AGC ATT TGG AT). Relative standard curve analysis was performed using the housekeeping gene B2M and unstimulated samples were used as a calibrator for fold-change.

Cells were stimulated with $100 \mathrm{ng} / \mathrm{mL}$ of TNC-TNF for $2 \mathrm{~h}$ and RNA was extracted using GENEzol Reagent (Geneaid) and cleaned using PureLink RNA Mini Kit (Invitrogen) according to the manufacturer's instructions. Libraries were prepared and sequenced at the Functional Genomics Center Zurich (Zurich, Switzerland). RNA-Seq data was processed through a standard workflow (https://github. com/csoneson/ARMOR), including read mapping against the mouse reference genome (Ensembl_GRCm38.90) using $\mathrm{STAR}^{50}$ and sorting/indexing with samtools ${ }^{51}$. Isoform-level expression estimation using salmon ${ }^{52}$ and gene-level differential expression (DE) analysis was performed using edge $\mathrm{R}^{53}$ with separate contrasts for each genotype (treated with TNC-TNF vs. untreated). Differential expression $t$-statistics used in the geneset analyses were computed using limma-voom ${ }^{54}$.

\section{Electron microscopy}

Cells were fixed in $2.5 \%$ glutaraldehyde in $0.1 \mathrm{M}$ cacodylate buffer and scraped from the plate. Transmission electron microscopy was performed by the University of Zurich, ZMB. 


\section{Statistical analysis}

All data is presented in mean \pm SEM. Figures were prepared in Illustrator CC 2015 (Adobe) and Prism 7 (GraphPad Software). Significance between genotypes and treatments was assessed by Student- $t$ test or two-way ANOVA with ${ }^{*} p<0.05,{ }^{* * *} p<0.01$, ${ }^{* * * * *} p<0.001$, ${ }^{* * * * * *} p<$ 0.0001 using Prism 7.

\section{Acknowledgements}

This research was supported by Hartmann Müller Stiftung, ZUNIV-FAN, Swiss National Science Project Grant, Novartis Foundation for Medical Biological Research, SPARKS XLP Trust Fund, Stiftung für wissenschaftliche Forschung an der Universität Zürich, EMDO Stiftung, Krebsliga Zürich. L.M.S. and L.V. were supported by the Forschungskredit Candoc (University of Zurich). W.W.W. is supported by the Clöetta Medical Research Fellow. The authors acknowledge the assistance and support of the Center for Microscopy and Image Analysis, University of Zurich for performing scanning electron microscopy experiments. H.W. is supported by DFG (grants WA1025/31-1 and TRR221 project B02. P.J.J. was supported by a Max Eder-Program grant from the Deutsche Krebshilfe (program \#111738), Deutsche José Carreras Leukämie-Stiftung (DJCLS R 12/22 and DJCLS 21R/2016), Else Kröner Fresenius Stiftung (2014_A185), from the Deutsche Forschungsgemeinschaft (DFG FOR 2036) and the Deutsche Konsortium für translationale Krebsforschung (DKTK) of the German Cancer Center (DKFZ). The authors thank Prof. Dr. C. Münz and A. Fontana for critical reading and discussions.

\section{Author details}

${ }^{1}$ Institute of Experimental Immunology, University of Zürich, Zürich, Switzerland. ${ }^{2}$ Institute of Pharmacology, University of Bern, Bern, Switzerland. ${ }^{3}$ III. Medizinische Klink, Klinikum rechts der Isar, Technische Universität München, Munich, Germany. ${ }^{4}$ UC Department of Pediatrics, Cincinnati Children's Hospital, Cincinnati, USA. ${ }^{5}$ Division of Molecular Internal Medicine, Department of Internal Medicine II, University Hospital Würzburg, Würzburg, Germany. ${ }^{6}$ Institute of Molecular Life Sciences and SIB Swiss Institute of Bioinformatics, University of Zürich, Zürich, Switzerland

\section{Author's contributions}

J.K., L.M.S., S.R. and W.W.W. designed the research. J.K., L.M.S., S.R., R.R., L.V. and W.W.W. performed the experiments. M.Y., P.J.J., H.W., M.R., R.A.M. and T.K. provided reagents and cell lines that made this study possible. J.K., L.M.S., S.R., M.R. and W.W.W. analyzed the data. J.K., L.M.S. and W.W.W. wrote the paper. J.K., S.R., L.V., M.Y., P.J.J., H.W., M.R. and T.K. gave feedback on the draft paper.

\section{Conflict of interest}

The authors declare that they have no conflict of interest.

\section{Publisher's note}

Springer Nature remains neutral with regard to jurisdictional claims in published maps and institutional affiliations.

Supplementary Information accompanies this paper at (https://doi.org/ 10.1038/s41419-019-1938-x).

Received: 28 August 2019 Accepted: 29 August 2019

Published online: 20 September 2019

\section{References}

1. Bell, J. H., Herrera, A. H., Li, Y. \& Walcheck, B. Role of ADAM17 in the ectodomain shedding of TNF-alpha and its receptors by neutrophils and macrophages. J. Leukoc. Biol. 82, 173-176 (2007).

2. Wicovsky, A. et al. Tumor necrosis factor receptor-associated factor-1 enhances proinflammatory TNF receptor-2 signaling and modifies TNFR1-TNFR2 cooperation. Oncogene 28, 1769-1781 (2009).

3. Dondelinger, Y. et al. MK2 phosphorylation of RIPK1 regulates TNF-mediated cell death. Nat. Cell Biol. 12, 147-150 (2017)
4. Menon, M. B. et al. p38MAPK/MK2-dependent phosphorylation controls cytotoxic RIPK1 signalling in inflammation and infection. Nat. Cell Biol. 76, 129-129 (2017).

5. Jaco, I. et al. MK2 phosphorylates RIPK1 to prevent TNF-induced cell death Mol. Cell 66, 698-710.e5 (2017).

6. Newton, $K$. et al. Activity of protein kinase RIPK3 determines whether cells die by necroptosis or apoptosis. Science 343, 1357-1360 (2014).

7. Newton, K. et al. RIPK1 inhibits ZBP1-driven necroptosis during development Nature 540, 129-133 (2016).

8. Murphy, J. M. et al. The pseudokinase MLKL mediates necroptosis via a molecular switch mechanism. Immunity 39, 443-453 (2013).

9. Grell, M. et al. The transmembrane form of tumor necrosis factor is the prime activating ligand of the $80 \mathrm{kDa}$ tumor necrosis factor receptor. Cell $\mathbf{8 3}$ 793-802 (1995)

10. Rothe, M., Pan, M. G., Henzel, W. J., Ayres, T. M. \& Goeddel, D. V. The TNFR2TRAF signaling complex contains two novel proteins related to baculoviral inhibitor of apoptosis proteins. Cell 83, 1243-1252 (1995).

11. Rauert, $\mathrm{H}$. et al. Membrane Tumor Necrosis Factor (TNF) Induces p100 Processing via TNF Receptor-2 (TNFR2). J. Biol. Chem. 285, 7394-7404 (2010).

12. Grell, M. et al. Induction of cell death by tumour necrosis factor (TNF) receptor 2, CD40 and CD30: a role for TNF-R1 activation by endogenous membraneanchored TNF. EMBO J. 18, 3034-3043 (1999).

13. Siegmund, D., Kums, J., Ehrenschwender, M. \& Wajant, H. Activation of TNFR2 sensitizes macrophages for TNFR1-mediated necroptosis. Cell Death Dis. 7, e2375-e2375 (2016)

14. Siegmund, D., Ehrenschwender, M. \& Wajant, H. TNFR2 unlocks a RIPK1 kinase activity-dependent mode of proinflammatory TNFR1 signaling. Cell Death Dis. 9, 921 (2018)

15. Yabal, M. et al. XIAP restricts TNF- and RIP3-dependent cell death and inflammasome activation. Cell Reports 7, 1796-1808 (2014).

16. Lawlor, K. E. et al. RIPK3 promotes cell death and NLRP3 inflammasome activation in the absence of MLKL. Nat. Commun. 6, 6282 (2015).

17. Wicki, S. et al. Loss of XIAP facilitates switch to TNFa-induced necroptosis in mouse neutrophils. Cell Death Dis. 7, e2422 (2016).

18. Bauernfeind, F. G. et al. Cutting edge: NF-kappaB activating pattern recognition and cytokine receptors license NLRP3 inflammasome activation by regulating NLRP3 expression. J. Immunol. 183, 787-791 (2009).

19. Martinon, F., Burns, K. \& Tschopp, J. The inflammasome: a molecular platform triggering activation of inflammatory caspases and processing of prolL-beta. Mol. Cell 10, 417-426 (2002)

20. Kayagaki, N. et al. Caspase-11 cleaves gasdermin D for non-canonical inflammasome signalling. Nature 526, 666-671 (2015).

21. Meunier, E. et al. Caspase-11 activation requires lysis of pathogen-containing vacuoles by IFN-induced GTPases. Nature 509, 366-370 (2014).

22. Lawlor, K. E. et al. XIAP loss triggers RIPK3- and caspase-8-Driven IL-1 $\beta$ activation and cell death as a consequence of TLR-MyD88-induced CIAP1-TRAF2 degradation. Cell Reports 20, 668-682 (2017)

23. Wong, W. W.-L. et al. CIAPs and XIAP regulate myelopoiesis through cytokine production in an RIPK1- and RIPK3-dependent manner. Blood 123, 2562-2572 (2014).

24. Chopra, M. et al. Exogenous TNFR2 activation protects from acute GvHD via host T reg cell expansion. J. Exp. Med. 213, 1881-1900 (2016)

25. Krippner-Heidenreich, $\mathrm{A}$. et al. Control of receptor-induced signaling complex formation by the kinetics of ligand/receptor interaction. J. Biol. Chem. 277, 44155-44163 (2002).

26. Schile, A. J., García-Fernández, M. \& Steller, H. Regulation of apoptosis by XIAP ubiquitin-ligase activity. Genes Dev. 22, 2256-2266 (2008).

27. Prokop, J. W. et al. Molecular modeling in the age of clinical genomics, the enterprise of the next generation. J. Mol. Model 23, 75 (2017).

28. Berger, S. B. et al. Cutting edge: RIP1 kinase activity is dispensable for norma development but is a key regulator of inflammation in SHARPIN-deficient mice. J. Immunol. 192, 5476-5480 (2014).

29. Wada, T. et al. Sustained elevation of serum interleukin-18 and its association with hemophagocytic lymphohistiocytosis in XIAP deficiency. Cytokine $\mathbf{6 5}$ 74-78 (2014)

30. Vince, J. E et al. Inhibitor of apoptosis proteins limit RIP3 kinase-dependent interleukin-1 activation. Immunity 36, 215-227 (2012).

31. Harlin, H., Reffey, S. B., Duckett, C. S., Lindsten, T. \& Thompson, C. B. Characterization of XIAP-deficient mice. Mol. Cell. Biol. 21, 3604-3608 (2001).

32. Polz, J. et al. Myeloid suppressor cells require membrane TNFR2 expression for suppressive activity. Immun., Inflamm. Dis. 2, 121-130 (2014). 
33. Zhao, $X$. et al. TNF signaling drives myeloid-derived suppressor cell accumulation. J. Clin. Invest 122, 4094-4104 (2012).

34. Schneider, P. et al. TWEAK can induce cell death via endogenous TNF and TNF receptor 1. Eur. J. Immunol. 29, 1785-1792 (1999).

35. Vince, J. E. et al. TWEAK-FN14 signaling induces lysosomal degradation of a CIAP1-TRAF2 complex to sensitize tumor cells to TNF. J. Cell Biol. 182, 171-184 (2008).

36. Henry, C. M. \& Martin, S. J. Caspase-8 acts in a non-enzymatic role as a scaffold for assembly of a pro-inflammatory "FADDosome" complex upon TRAlL stimulation. Mol. Cell 65, 715-729.e5 (2017).

37. Najjar, M. et al. RIPK1 and RIPK3 kinases promote cell-death-independent inflammation by toll-like receptor 4. Immunity 1-50 (2016). https://doi.org/ 10.1016/j.immuni.2016.06.007

38. Chirieleison, S. M. et al. Nucleotide-binding oligomerization domain (NOD) signaling defects and cell death susceptibility cannot be uncoupled in Xlinked inhibitor of apoptosis (XIAP)-driven inflammatory disease. J. Biol. Chem. 292, 9666-9679 (2017).

39. Damgaard, R. B. et al. Disease-causing mutations in the XIAPBIR2 domain impair NOD2-dependent immune signalling. EMBO Mol. Med. 5, 1278-1295 (2013).

40. Damgaard, R. B. et al. The ubiquitin ligase XIAP recruits LUBAC for NOD2 signaling in inflammation and innate immunity. Mol. Cell 46, 746-758 (2012).

41. Ammann, S. et al. A new functional assay for the diagnosis of $X$-linked inhibitor of apoptosis (XIAP) deficiency. Clin. Exp. Immunol. 176, 394-400 (2014).

42. Filipovich, A. H., Zhang, K., Snow, A. L. \& Marsh, R. A. X-linked lymphoproliferative syndromes: brothers or distant cousins? Blood 116, 3398-3408 (2010).
43. Schmid, J. P. et al. Clinical similarities and differences of patients with X-linked lymphoproliferative syndrome type 1 (XLP-1/SAP deficiency) versus type 2 (XLP-2/XIAP deficiency). Blood 117, 1522-1529 (2011).

44. Marsh, R. A. et al. Allogeneic hematopoietic cell transplantation for XIAP deficiency: an international survey reveals poor outcomes. Blood 121, 877-883 (2013).

45. Malireddi, R. K. S. et al. TAK1 restricts spontaneous NLRP3 activation and cell death to control myeloid proliferation. J. Exp. Med. 215, 1023-1034 (2018).

46. Moulin, M. et al. IAPs limit activation of RIP kinases by TNF receptor 1 during development. EMBO J. 31, 1679-1691 (2012).

47. Olayioye, M. A. et al. XIAP-deficiency leads to delayed lobuloalveolar development in the mammary gland. Cell Death Differ. 12, 87-90 (2004).

48. Peschon, J. J. et al. TNF receptor-deficient mice reveal divergent roles for p55 and p75 in several models of inflammation. J. Immunol. 160, 943-952 (1998).

49. Fick, A., Wyzgol, A.\& Wajant, H. Production, purification, and characterization of scFv TNF ligand fusion proteins. Methods Mol. Biol. 907, 597-609 (2012).

50. Dobin, A. et al. STAR: ultrafast universal RNA-seq aligner. Bioinformatics 29, 15-21 (2012).

51. Li, H. et al. The Sequence Alignment/Map format and SAMtools. Bioinformatics 25, 2078-2079 (2009)

52. Patro, R., Duggal, G., Love, M. I., Irizarry, R. A. \& Kingsford, C. Salmon provides fast and bias-aware quantification of transcript expression. Nat. Publ. Group 14, 417-419 (2017)

53. Robinson, M. D., McCarthy, D. J. \& Smyth, G. K. edgeR: a Bioconductor package for differential expression analysis of digital gene expression data. Bioinformatics 26, 139-140 (2009).

54. Law, C. W., Chen, Y., Shi, W. \& Smyth, G. K. voom: precision weights unlock linear model analysis tools for RNA-seq read counts. Genome Biol. 15, R29 (2014). 